\title{
Pain in patients with multiple sclerosis: a complex assessment including quantitative and qualitative measurements provides for a disease-related biopsychosocial pain model
}

\author{
This article was published in the following Dove Press journal: \\ Journal of Pain Research \\ | August 201। \\ Number of times this article has been viewed
}

\author{
Dominik Michalskil,* \\ Stefanie Liebigl,* \\ Eva Thomae ${ }^{1,2}$ \\ Andreas $\mathrm{Hinz}^{3}$ \\ Florian Then Bergh ${ }^{1,2}$ \\ 'Department of Neurology, \\ ${ }^{2}$ Translational Centre for Regenerative \\ Medicine (TRM), ${ }^{3}$ Department of \\ Medical Psychology and Medical \\ Sociology, University of Leipzig, \\ Leipzig, Germany \\ *These authors contributed equally
}

\begin{abstract}
Background: Pain of various causes is a common phenomenon in patients with Multiple Sclerosis (MS). A biopsychosocial perspective has proven a useful theoretical construct in other chronic pain conditions and was also started in MS. To support such an approach, we aimed to investigate pain in MS with special emphasis on separating quantitative and qualitative aspects, and its interrelation to behavioral and physical aspects.
\end{abstract}

Materials and methods: Pain intensity (NRS) and quality (SES) were measured in 38 consecutive outpatients with MS (mean age, $42.0 \pm 11.5$ years, $82 \%$ women). Pain-related behavior (FSR), health care utilization, bodily complaints (GBB-24) and fatigue (WEIMuS) were assessed by questionnaires, and MS-related neurological impairment by a standardized neurological examination (EDSS).

Results: Mean pain intensity was 4.0 (range, 0-10) and mean EDSS 3.7 (range, 0-8) in the overall sample. Currently present pain was reported by $81.6 \%$ of all patients. Disease duration and EDSS did not differ between patients with and without pain and were not correlated to quality or intensity of pain. Patients with pain had significantly higher scores of musculoskeletal complaints, but equal scores of exhaustion, gastrointestinal and cardiovascular complaints. Pain intensity correlated only with physical aspects, whereas quality of pain was additionally associated with increased avoidance, resignation and cognitive fatigue.

Conclusion: As in other conditions, pain in MS must be assessed in a multidimensional way. Further research should be devoted to adapt existing models to a MS-specific model of pain.

Keywords: pain intensity, quality of pain, pain-related behavior, bodily complaints, multiple sclerosis

\section{Introduction}

Multiple sclerosis (MS) is a chronic immune-mediated disease of the central nervous system that often leads to long-term physical disability. ${ }^{1}$ Throughout the course of the disease, pain is a common, varying symptom and a significant problem. ${ }^{2}$ In $23 \%$ of patients with MS, pain was found as part of the presenting symptoms at disease onset. ${ }^{3}$ Previous studies have reported remarkable pain prevalences up to $79.4 \%$ and $92 \%$ in patients with MS. ${ }^{4,5}$ Pain in MS patients can be caused directly by inflammatory attack on afferent fiber tracts, or indirectly by various sequelae of MS (eg, limb paresis, extrapyramidal or cerebellar syndromes, bladder dysfunction), ${ }^{1}$ leading to increased muscle tone with spasms, postural abnormalities, or urinary tract infections. ${ }^{2,6}$ In addition, drug treatment is a potential contributor to pain. ${ }^{6,7} \mathrm{On}$
Correspondence: Dominik Michalsk University of Leipzig, Department of Neurology, Liebigstr. 20, 04103 Leipzig, Germany Tel +49 34I 9724206 Fax +4934I 9724257 Email dominik.michalski@medizin. uni-leipzig.de 
the other hand, pain in MS influences many aspects of daily living ${ }^{2,8,9}$ (eg, important domains of health-related quality of life $)^{10}$ and physical activity. ${ }^{11,12}$ The consequences of pain in MS should be assessed with a focus on psychosocial aspects - an issue that has received insufficient attention in numerous previous studies. ${ }^{2,13}$ The available data suggest that depression, pain behavior, health care utilization, coping behavior, and beliefs might be important determinants of pain. ${ }^{2,6,13-16}$ In other painful conditions - such as low back pain - biopsychosocial models, which describe interactions of bodily pain with cognitive, behavioral, and social variables, have proven useful in understanding the complex conditions of the disorder and guiding interventions. ${ }^{17-20}$ Kerns et $\mathrm{al}^{6,13}$ proposed a multidimensional model for pain in MS; amongst others, behavioral aspects have received more attention in this model. For example, muscle weakness can contribute to more generalized deconditioning and increased musculoskeletal complaints; these changes, in combination with fear of pain, contribute to avoidance behavior with a consecutive worsening of muscle weakness and thus additional pain. ${ }^{6}$ The usefulness of this analytical process in MS patients was underscored by a recent study by Motl et al, ${ }^{21}$ who focused on behavioral aspects, especially physical activity. They found that MS patients who were more physically active had lower scores of pain along with reduced depression and fatigue, as well as improved self-esteem. Comparable data were also presented by Turner et al. ${ }^{22}$ Another investigation showed a significant association of pain beliefs and coping strategies with pain intensity scores in patients with MS. ${ }^{15}$ Generally, previous studies in MS patients often assessed pain in a dichotomous way, providing the proportion of patients with and without pain. ${ }^{9-12}$ This was sometimes supplemented with quantifying pain intensity using rating scales. ${ }^{5,9-12,16,23}$ One aspect that has been incompletely addressed - especially in the field of neuropathic pain - is recording both pain intensity and quality of pain. ${ }^{24}$

The aims of the present study were (1) to investigate both pain intensity and quality of pain in MS patients predominantly in a quantitative modality - and (2) to analyze their interrelations to physical (bodily complaints, fatigue) and behavioral factors (pain-related behavior, health care utilization), exploring the components of a biopsychosocial model applicable to MS.

\section{Materials and methods Study design and population}

In a prospective setting, unselected consecutive outpatients attending the Department of Neurology of the University of
Leipzig in 2007 with an established diagnosis of MS were asked to participate in a survey using a standardized paperand-pencil questionnaire. Data from 49 participants were available; 38 of these subjects provided information about pain and were analyzed.

\section{Assessments}

Pain intensity (NRS) was assessed with a numerical rating scale (scaled from 0 to 10$)^{25}$ addressing the average pain, which is associated with MS according to the patient's point of view. Thereby, 0 represents no pain and 10 the most painful sensation imaginable. In addition, the lowest and highest intensity of pain were recorded.

Quality of pain (SES) was measured by the pain sensation scale. ${ }^{26}$ This tool contains 24 adjectives of pain sensation in a questionnaire; each of them is scaled from 1 to 4 . Fourteen items comprise the affective dimension, and ten items contribute to the sensory dimension.

Bodily complaints (GBB-24) were measured using the short version of the Giessen-subjective complaints list. ${ }^{27}$ In this questionnaire patients rate a list of 24 complaints according to currently perceived severity from 0 to 4 . The items are attributed equally to four dimensions: exhaustion, gastrointestinal complaints, musculoskeletal complaints and cardiovascular complaints. The total score of complaints equals the sum of the four dimensions. The GBB-24 is one of the most commonly used complaints lists in Germany and was standardized using the German population. ${ }^{28}$

Fatigue (WEIMuS) was assessed by the Würzburg fatigue inventory for multiple sclerosis as described by Flachenecker et al. ${ }^{29}$ In this questionnaire 17 items (scaled from 0 to 4 ) are listed, resulting in two dimensions: cognitive fatigue and physical fatigue. The scale combines items from the Fatigue Severity Scale (FSS) and the Modified Fatigue Impact Scale (MFIS) simplifying the partly overlapping scales; it has been validated in a German MS population and found to reflect all aspects covered when administering the separate scales. ${ }^{29}$

Pain-related behavior (FSR) was measured with the dimensions avoidance, resignation and distraction of the Questionnaire on Pain Regulation; in each case eight items (scaled from 1 to 7 ) generate one dimension. ${ }^{30}$

Health care utilization reflects the self-reported number of MS-related physician consultations, physician changes, days off work, physical therapies, days of hospitalization and surgical interventions within the last 12 months.

The severity of neurological impairment due to MS was quantified by the Expanded Disability Status Scale (EDSS), ${ }^{31}$ 
the most common scale to measure impairment in MS patients ranging from 0 to 10 in 0.5 increments, ${ }^{32}$ around the time of completing the questionnaire.

For all measurements, higher values correspond to higher intensity of each respective dimension.

\section{Statistical analyses}

Questionnaire scores (GBB-24, SES, WEIMuS and FSR) were calculated as rounded means with a tolerance of one missing item per scale. Descriptive statistics were applied for sample descriptions. For differences between two groups, the Kolmogorov-Smirnov test was used. Analysis of Variance (ANOVA) was applied for differences between more than two groups, followed by the Duncan test for multiple comparisons. Relations between variables were analyzed using Pearson's correlations. Data are given as mean \pm standard deviation, unless otherwise indicated. All calculations were performed with SPSS (v 18.0; SPSS Inc, an IBM Company, Chicago, IL). A $P$-value $<0.05$ was considered statistically significant.

\section{Results}

The present sample of 38 patients with MS consisted of seven men (18.4\%) and 31 women (81.6\%). Patients' mean age was $42.0 \pm 11.5$ years (range, 21-69) and all were native Germans. The mean disease duration was $8.6 \pm 7.3$ years (range, 1-26). The clinical course of MS was relapsing-remitting in 24 (RR; 63.2\%), secondary progressive in seven (SP; $18.4 \%$ ), and primary progressive in five patients (PP; 13.2\%). Two patients (5.2\%) suffered from a clinical isolated syndrome of CNS demyelination (CIS). Due to the small number, the latter group was not used for analysis of differences according to the clinical course.

\section{Pain intensity and quality of pain}

Table 1 gives an overview on pain intensity and quality of pain as well as the physical and behavioral aspects. There was no significant difference between men and women for pain intensity (men, $3.9 \pm 3.0$; women, $4.1 \pm 2.6 ; P=1.000$ ), nor for sensory (men, $21.0 \pm 8.9$; women, $16.4 \pm 6.5 ; P=0.726$ ) or affective dimensions of pain quality (men, $29.3 \pm 13.0$; women, $26.8 \pm 10.7 ; P=0.898)$. Age did not correlate with pain intensity $(r=0.14 ; P=0.390)$, sensory $(r=0.18$; $P=0.328)$ or affective $(r=0.20 ; P=0.281)$ dimensions of pain quality. Course of MS (RR, SP and PP) did not differ with respect to pain intensity, affective or sensory quality of pain $(P=0.721 ; P=0.243 ; P=0.413$, respectively $)$. Disease duration was not correlated with pain intensity $(r=0.24$; $P=0.143)$, affective $(r=0.26 ; P=0.174)$ or sensory $(r=0.14 ; P=0.470)$ dimensions of pain quality.

Table I Pain intensity and quality, as well as physical and behavioral aspects in the overall sample

\begin{tabular}{|c|c|c|c|c|}
\hline & & Mean & Standard deviation & Range \\
\hline \multirow[t]{4}{*}{ Pain } & Pain intensity & 4.0 & 2.6 & $0-10$ \\
\hline & Pain quality & & & \\
\hline & Affective dimension & 27.1 & 10.9 & $|4-5|$ \\
\hline & Sensory dimension & 17.0 & 6.9 & $9-33$ \\
\hline \multirow[t]{10}{*}{ Physical aspects } & EDSS & 3.7 & 2.4 & $0-8$ \\
\hline & Bodily complaints & & & \\
\hline & Exhaustion & 12.0 & 5.4 & $0-21$ \\
\hline & Gastrointestinal complaints & 2.9 & 2.9 & $0-12$ \\
\hline & Musculoskeletal complaints & 10.6 & 4.2 & $3-19$ \\
\hline & Cardiovascular complaints & 3.9 & 3.3 & $0-12$ \\
\hline & Total score & 29.4 & 12.5 & $6-55$ \\
\hline & Fatigue & & & \\
\hline & Cognitive fatigue & 13.3 & 9.4 & $0-36$ \\
\hline & Physical fatigue & 17.5 & 8.6 & $0-31$ \\
\hline \multirow[t]{11}{*}{ Behavioral aspects } & Pain-related behavior & & & \\
\hline & Avoidance & 29.4 & 10.3 & $8-47$ \\
\hline & Resignation & 31.9 & 10.5 & $9-50$ \\
\hline & Distraction & 28.4 & 9.6 & $|2-5|$ \\
\hline & Health care utilization (number within the last 12 months) & & & \\
\hline & Physician consultations & 9.1 & 5.3 & $2-25$ \\
\hline & Physician changes & 0.2 & 0.5 & $0-2$ \\
\hline & Days off work & 58.8 & 112.8 & $0-365$ \\
\hline & Physical therapies & 45.0 & 68.8 & $0-260$ \\
\hline & Days of hospitalization & 6.7 & 10.2 & $0-42$ \\
\hline & Surgical interventions & 0.0 & 0.0 & $0-0$ \\
\hline
\end{tabular}




\section{Frequency of pain}

Among 38 patients, 31 reported pain intensity above zero. This results in $81.6 \%$ of patients experiencing pain at the time of completing the questionnaire. Patients with pain reported MS-related "highest pain intensity" of 7.1 \pm 2.0 (range, 2-10) and "lowest pain intensity" of $2.0 \pm 1.8$ (range, $0-7$ ).

\section{Differences between MS patients with pain vs no pain}

MS patients with pain and those who had no pain were of equal age (pain, $42.4 \pm 11.4$ years; no pain, $40.0 \pm 12.4$ years; $P=0.871)$, had comparable neurological impairment (EDSS: pain, $3.8 \pm 2.3$; no pain, $3.1 \pm 3.1 ; P=0.556$ ) and disease duration (pain, $8.9 \pm 7.6$ years; no pain, $7.3 \pm 6.2$ years; $P=0.885$ ). The direct comparisons between the pain and no pain group concerning bodily complaints and fatigue are shown in Figure 1. Those concerning painrelated behavior and health care utilization are illustrated in Figure 2. Significant differences are only apparent for musculoskeletal complaints $(P=0.003)$ and total score of complaints $(P=0.033)$, with higher values for patients with pain. Patients with pain tended to increased avoidance and resignation, and attended fewer physical therapy sessions and had increased days off work, though with no statistical significance $(P=0.527 ; P=0.265$, respectively).

\section{Relationship between pain and physical as well as behavioral aspects}

Table 2 shows the interrelation of assessed parameters in terms of a correlation matrix. Pain intensity correlated significantly with musculoskeletal complaints and consecutively with total score of bodily complaints. A further significant coefficient was found for physical fatigue. Interestingly, there was no significant interrelation to behavioral aspects. The affective dimension of quality of pain was associated with exhaustion and total score of bodily complaints, and furthermore physical fatigue. Concerning behavioral aspects, the affective dimension was significantly correlated with avoidance and resignation. The sensory dimension of quality of pain was significantly associated with physical aspects including all types of bodily complaints and fatigue, but not with severity of neurological impairment (EDSS). A further interrelation was found between the sensory dimension of pain and resignation. Summarizing pain concerning different dimensions, the qualitative aspect of pain was substantially associated with pain-related behavior in contrast to the quantitative dimension. Moreover, pain intensity and quality of pain did not significantly interact with health care utilization (bottom of Table 2).

\section{Discussion}

Eighty-one percent of our unselected sample of outpatients with MS suffered from pain at the time of completing the questionnaire. This proportion is comparable with previous studies (eg, 79.4\%), ${ }^{23}$ although higher $(92 \%)^{5}$ and lower percentages have been reported as well (eg, $42.9 \%$ and $66 \%)^{4,12}$

Focusing on physical aspects of MS, the means for bodily complaints are increased nearly two-fold for exhaustion, musculoskeletal complaints, and total score of complaints,

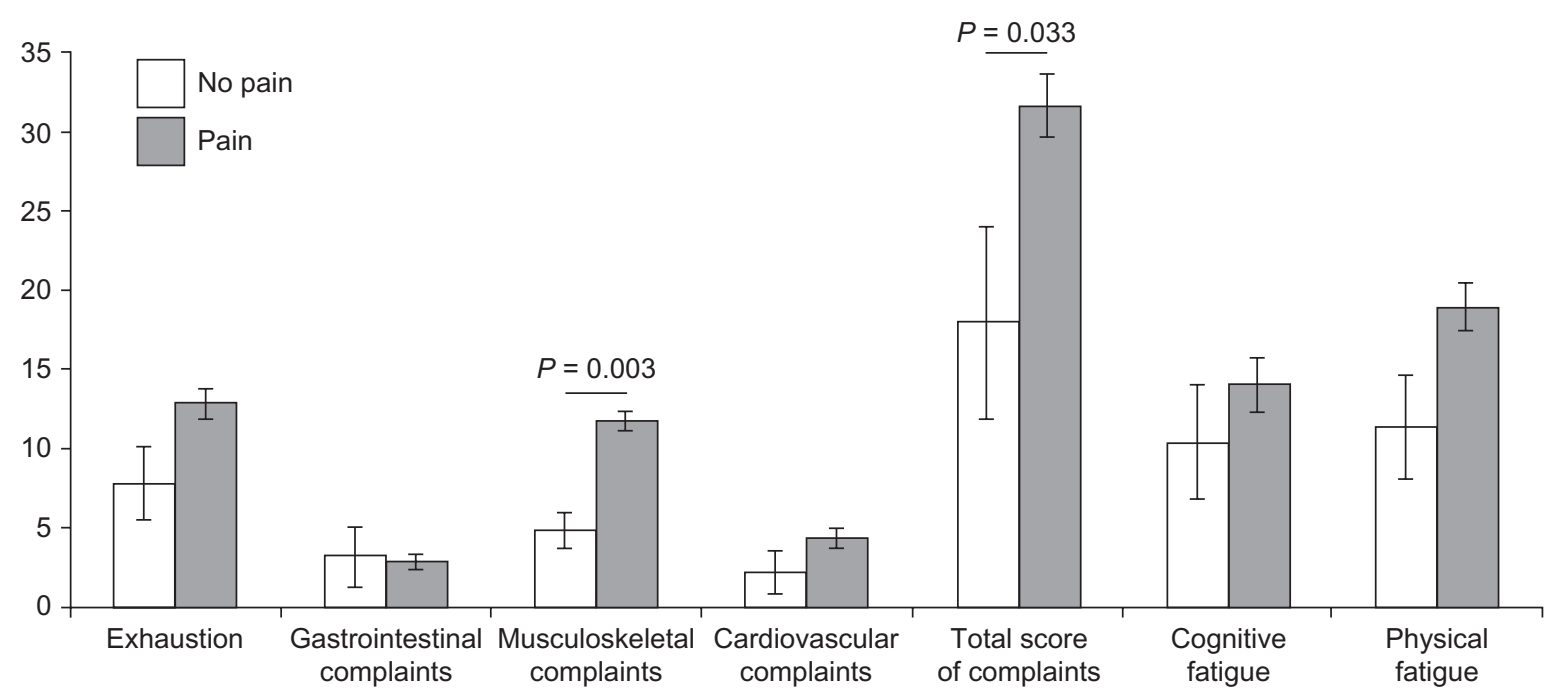

Figure I Comparison of physical aspects (bodily complaints and fatigue) between MS patients with and with no pain, displayed as means in bars and standard errors of means in lines. 


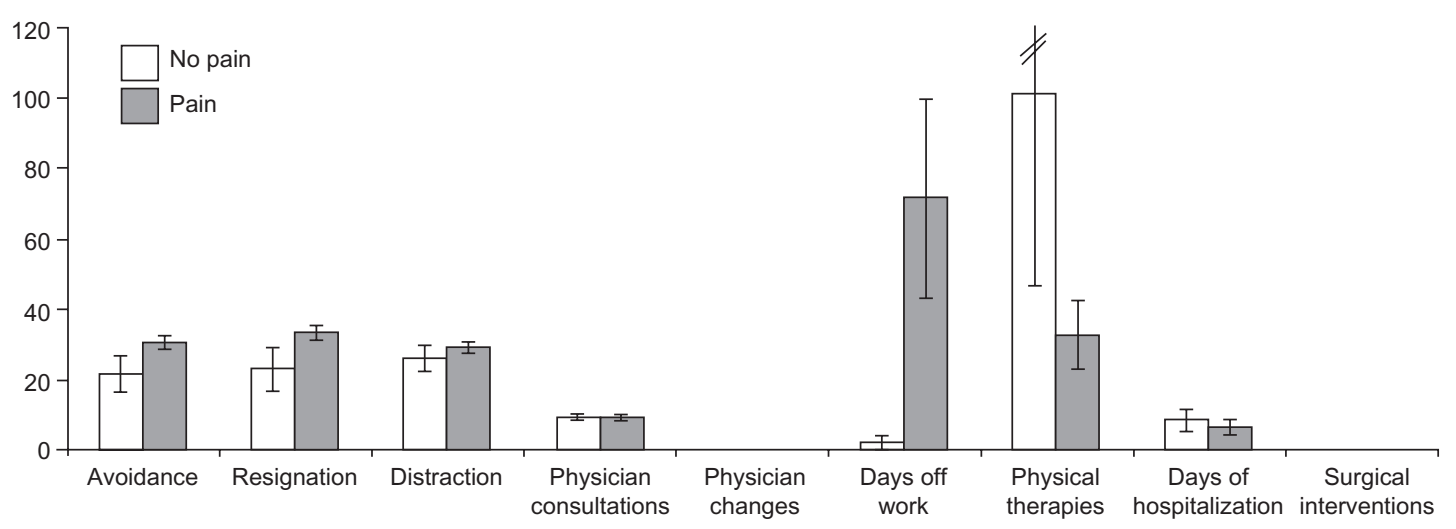

Figure 2 Comparison of behavioral aspects (pain-related behavior and health care utilization) between MS patients with and with no pain, displayed as means in bars and standard errors of means in lines.

as compared to the German general population (means: exhaustion, 3.97; musculoskeletal complaints, 5.35; and total score of complaints, 14.03). ${ }^{28}$ Cognitive and physical fatigue are nearly equal to values that have been described by Flachenecker et $\mathrm{al}^{29}$ who validated the WEIMuS scale in 67 patients with MS, which resulted in mean scores of 14.7 for cognitive fatigue and 19.9 for physical fatigue. Pain-related behavior in our sample was remarkably comparable to a group described as "back pain with low competence-estimation", which was composed of 73 patients and listed in the user manual of the questionnaire (means for comparison: avoidance, 28.34; resignation, 33.21; and distraction, 29.30). ${ }^{30}$
The fact that pain - irrespective of pain intensity or quality - did not correlate with age, disease duration, or severity of impairment due to MS (EDSS) confirms and extends earlier findings, $3,5,10,12,16$ though two other studies did obtain positive correlations to age and disease duration, ${ }^{4}$ as well as to EDSS. ${ }^{4,11}$ However, differing pain assessments and aims might be possible explanations. In detail, Solaro et $\mathrm{al}^{4}$ recorded presence of neuropathic, somatic, or visceral pain without evaluation of intensity. In contrast, Ehde et $\mathrm{al}^{11}$ used an 11-point rating scale for pain assessment in a large community sample of MS, but focused on pain-related activity interference, in particular by utilizing an interference numeric

Table 2 Correlation matrix of pain (intensity, affective and sensory dimension), physical and behavioral aspects in patients with MS

\begin{tabular}{|c|c|c|c|c|}
\hline & & $\begin{array}{l}\text { Pain intensity } \\
r(P \text {-value })\end{array}$ & $\begin{array}{l}\text { Affective dimension } \\
\text { of pain quality } \\
r \text { (P-value) }\end{array}$ & $\begin{array}{l}\text { Sensory dimension } \\
\text { of pain quality } \\
r \text { ( } P \text {-value })\end{array}$ \\
\hline \multirow[t]{10}{*}{ Physical aspects } & EDSS & $0.23(0.158)$ & $0.09(0.639)$ & 0.01 (0.978) \\
\hline & Bodily complaints & & & \\
\hline & Exhaustion & $0.32(0.052)$ & $0.56(0.00 I)$ & $0.4 I(0.02 I)$ \\
\hline & Gastrointestinal complaints & $0.06(0.747)$ & $0.29(0.125)$ & $0.40(0.025)$ \\
\hline & Musculoskeletal complaints & $0.65(0.000)$ & $0.35(0.062)$ & $0.49(0.006)$ \\
\hline & Cardiovascular complaints & $0.24(0.152)$ & $0.17(0.362)$ & $0.49(0.006)$ \\
\hline & Total score & $0.43(0.007)$ & $0.48(0.008)$ & $0.56(0.001)$ \\
\hline & Fatigue & & & \\
\hline & Cognitive fatigue & $0.19(0.246)$ & $0.24(0.202)$ & $0.56(0.001)$ \\
\hline & Physical fatigue & $0.33(0.044)$ & $0.49(0.006)$ & $0.53(0.002)$ \\
\hline \multirow[t]{11}{*}{ Behavioral aspects } & Pain-related behavior & & & \\
\hline & Avoidance & $0.12(0.496)$ & $0.58(0.001)$ & $0.35(0.053)$ \\
\hline & Resignation & $0.07(0.695)$ & $0.46(0.010)$ & $0.36(0.048)$ \\
\hline & Distraction & $-0.01(0.950)$ & $-0.29(0.125)$ & $0.04(0.853)$ \\
\hline & Health care utilization & & & \\
\hline & Physician consultations & $0.15(0.431)$ & $0.41(0.055)$ & $0.22(0.3 \mid 4)$ \\
\hline & Physician changes & $0.09(0.648)$ & $0.14(0.540)$ & $0.08(0.742)$ \\
\hline & Days off work & $0.36(0.102)$ & $-0.21(0.416)$ & $0.22(0.385)$ \\
\hline & Physical therapies & $-0.19(0.330)$ & $-0.03(0.904)$ & $-0.15(0.507)$ \\
\hline & Days of hospitalization & $0.00(0.984)$ & $-0.13(0.566)$ & $-0.10(0.644)$ \\
\hline & Surgical interventions & - & - & - \\
\hline
\end{tabular}

Notes: $r$, Pearson's coefficient; $P$-value, significance. 
rating scale. Notwithstanding these heterogeneous findings, the lack of gender-related differences in pain intensity and quality has been consistently reported. , $3,11,12^{2}$

With respect to a biopsychosocial perspective of pain in MS, it is remarkable that pain was not significantly correlated with EDSS or disease duration. This underscores the need to incorporate physical aspects apart from solely neurological impairment and psychosocial issues. Musculoskeletal complaints were significantly different between the pain and no pain group. This, together with the known predominant pain localization of MS patients - in legs and lower back (74.6\% and $59.3 \%)$ and neck and shoulders $(51.7 \% \text { and } 49.2 \%)^{12}-$ primarily reflects chronic postural misadjustments. However, the recognition of rather prevalent associations with rheumatoid arthritis and recently with the TNF receptor associated periodic syndrome (TRAPS) ${ }^{33}$ add further potentially treatable physical factors. With respect to psychosocial issues, Kerns et al ${ }^{6,13}$ especially emphasized cognitive, behavioral and social issues in their biopsychosocial pain model. In line with this extended perspective, Arnett et al ${ }^{14}$ recently presented a model of MS-specific depression and thereby pointed out the inconsistent association to physical disability, but supported the use of psychosocial variables (eg, coping and social support). In the behavioral domain, the tendency of increased days off work in patients with pain - which has been found in this study and also by Ehde et al ${ }^{12}$ - confirms its socioeconomic consequences in MS.

The differentiated analysis of associations between pain intensity and quality as well as physical and behavioral aspects was performed in the whole sample size, thus representing a quantitative assessment. Overall, more significant coefficients were found for quality of pain when compared with pure pain intensity - this underscores the point of view that pain in MS must be measured by using such differentiated scores, analogous to peripheral neuropathic pain. ${ }^{24}$ More specifically, the affective and sensory dimensions of quality of pain had an impact on pain-related behavior, while increasing pain led to augmented avoidance and resignation. The propensity to avoidance is comparable to the mechanisms attributed to chronic low back pain, ${ }^{18}$ and suggests that beliefs ${ }^{19}$ and coping strategies ${ }^{15,20}$ similarly affect pain in MS patients. An increased affective pain dimension, linked to exhaustion and total score of complaints, might lead to avoidance behavior, bodily deconditioning and thus more pain. This postulation proves similar to the model of Kerns et al. ${ }^{6,13}$ The additional effect of fatigue due to pain and behavioral avoidance can be inferred from the correlation of both affective and sensory pain dimensions to fatigue. Such interactions of pain, avoidance and fatigue were also addressed in the biopsychosocial model of Kerns et al, ${ }^{6}$ and the connection of pain and fatigue in general has been noted previously. ${ }^{34}$ Consistent with this direction, and potentially adding another therapeutic opportunity, is the nonsignificant negative correlation of affective pain and distraction. Exaggerated pain perception due to fear of pain, which can be caused by increased interoception, might prevent patients from seeking distraction from painful sensations. ${ }^{17}$ Furthermore, bodily deconditioning and social isolation reduce joyful experience, in turn contributing to affective disorders such as depression. .,14 $^{-14}$

The present study had some limitations: First, the sample size was relatively small, affecting the validity of the study conclusions. Despite this limitation, our study revealed some significant interactions in a biopsychosocial approach of pain in MS, which might be useful in exploring a MS-specific pain model. According to a multifactorial perspective, affective components like depression were not addressed, though these issues of MS are garnering increasing interest in recent years. ${ }^{14}$ However, this study was clearly aimed at pain intensity and quality in relation to physical and behavioral factors. Focusing on pain assessment in general, the use of NRS has sometimes been criticized, ${ }^{35}$ but sufficiently evaluated tools for multidimensional pain measurement are still lacking.

In summary, physical activity should be encouraged in MS patients with pain in order to prevent bodily deconditioning, secondary fatigue, social isolation and increased pain sensation. This model is supported by the finding that MS patients who are more physically active suffer less from fatigue, pain and depression. ${ }^{21,22}$ However, neurological impairment due to MS - eg, spasms or postural abnormalities - often leads to patients avoiding activity and must be taken into account. The therapeutic challenge is to integrate information about individual physical etiology with the analysis of behavioral aspects in an effort to intercept self-amplifying pain mechanisms. Cognitive-behavioral interventions are expected to be useful in this approach. ${ }^{15,16}$

\section{Acknowledgments}

We thank Silvia Ceballos, Department of Neurology, University of Leipzig, Germany, for helping to organize this survey. This study was conducted at the authors' institutions and was made possible in part by funding from the German Federal Ministry of Education and Research (BMBF, PtJ-Bio, 0313909) to E Thomae and F Then Bergh. 


\section{Disclosure}

The authors have declared no conflicts of interest in this work.

\section{References}

1. Noseworthy JH, Lucchinetti C, Rodriguez M, Weinshenker BG. Multiple Sclerosis. N Engl J Med. 2000;343(13):938-952.

2. Ehde DM, Osborne TL, Jensen MP. Chronic pain in persons with multiple sclerosis. Phys Med Rehabil Clin N Am. 2005;16(2):503-512.

3. Stenager E, Knudsen L, Jensen K. Acute and chronic pain syndromes in multiple sclerosis. Acta Neurol Scand. 1991;84(3):197-200.

4. Solaro C, Brichetto G, Amato MPl, et al; PaIMS Study Group. The prevalence of pain in multiple sclerosis. A multicenter cross-sectional study. Neurology. 2004;63(5):919-921.

5. Hirsh AT, Turner AP, Ehde DM, Haselkorn JK. Prevalence and impact of pain in multiple sclerosis: physical and psychologic contributors. Arch Phys Med Rehabil. 2009;90(4):646-651.

6. Kerns RD, Kassirer M, Otis J. Pain in multiple sclerosis: a biopsychosocial perspective. J Rehab Res Dev. 2002;39(2):225-232.

7. Pöllmann W, Erasmus LP, Feneberg W, Then Bergh F, Straube A. Interferon beta but not glatiramer acetate therapy aggravates headaches in MS. Neurology. 2002;59(4):636-639.

8. Benrud-Larson LM, Wegener ST. Chronic pain in neurorehabilitation populations: prevalence, severity and impact. NeuroRehabilitation. 2000;14(3):127-137.

9. Archibald CJ, McGrath PJ, Ritvo PG, et al. Pain prevalence, severity and impact in a clinic sample of multiple sclerosis patients. Pain. 1994; 58(1):89-93.

10. Grasso MG, Clemenzi A, Tonini A, et al. Pain in multiple sclerosis: a clinical and instrumental approach. Mult Scler. 2008;14(4):506-513.

11. Ehde DM, Gibbons LE, Chwastiak L, Bombardier CH, Sullivan MD, Kraft GH. Chronic pain in a large community sample of persons with multiple sclerosis. Mult Scler. 2003;9(6):605-611.

12. Ehde DM, Osborne TL, Hanley MA, Jensen MP, Kraft GH. The scope and nature of pain in persons with multiple sclerosis. Mult Scler. 2006;12(5):629-638.

13. Kerns RD. Psychosocial aspects of pain. Int J Mult Scler Care. 2000; 2(4):35-38.

14. Arnett PA, Barwick FH, Beeney JE. Depression in multiple sclerosis: review and theoretical proposal. J Int Neuropsychol Soc. 2008;14(5): 691-724.

15. Douglas C, Wollin JA, Windsor C. Biopsychosocial correlates of adjustment to pain among people with multiple sclerosis. Clin J Pain. 2008;24(7):559-567.

16. Osborne TL, Jensen MP, Ehde DM, Hanley MA, Kraft G. Psychosocial factors associated with pain intensity, pain-related interference, and psychological functioning in persons with multiple sclerosis and pain. Pain. 2007;127(1-2):52-62.

17. Waddel G. The back pain revolution. Edinburgh, Scotland: Churchill Livingstone; 2004.
18. Poiraudeau S, Rannou F, Baron G, et al. Fear-avoidance beliefs about back pain in patients with subacute low back pain. Pain. 2006;124(3): 305-311.

19. Turner J, Jensen MP, Romano JM. Do beliefs, coping, and catastrophizing independently predict functioning in patients with chronic pain? Pain. 2000;85(1-2):115-125.

20. Jensen MP, Turner JA, Romano JM, Karoly P. Coping with chronic pain: a critical review of the literature. Pain. 1991;47(3):249-283.

21. Motl RW, McAuley E, Snook EM, Gliottoni RC. Physical activity and quality of life in multiple sclerosis: intermediary roles of disability, fatigue, mood, pain, self-efficacy and social support. Psychol Health Med. 2009;14(1):111-124.

22. Turner AP, Kivlahan DR, Haselkorn JK. Exercise and quality of life among people with multiple sclerosis: looking beyond physical functioning to mental health and participation in life. Arch Phys Med Rehabil. 2009;90(3):420-428.

23. Svendsen KB, Jensen TS, Overvad K, Hansen HJ, KochHenriksen N, Bach FW. Pain in patients with multiple sclerosis. Arch Neurol. 2003;60(8):1089-1094.

24. Cruccu G, Anand P, Attal N, et al. EFNS guidelines on neuropathic pain assessment. Eur J Neurol. 2004;11(3):153-162.

25. Price DD, Bush FM, Long S, Harkins SW. A comparison of pain measurement characteristics of mechanical visual analogue and simple numerical rating scales. Pain. 1994;56(2):217-226.

26. Geissner E. The pain sensation scale (SES). Göttingen, Germany: Hogrefe; 1996.

27. Brähler E, Hinz A, Scheer JW. The Giessen-subjective complaints list (GBB-24). Bern, Switzerland: Huber; 2008

28. Brähler E, Schumacher J, Brähler C. First standardization of the short version of the Giessen-subjective complaints list GBB-24 in re-unified Germany. Psychother Psychosom Med Psychol. 2000;50(1):14-21.

29. Flachenecker P, Müller G, König H, Meissner H, Toyka K, Rieckmann P. Fatigue and multiple sclerosis. Nervenarzt. 2006;77(2): $165-174$.

30. Schermelleh-Engel K. Questionnaire on pain regulation (FSR). Frankfurt, Germany: Swets and Zeitlinger; 1995.

31. Kurtzke JF. Rating neurologic impairment in multiple sclerosis: an expanded disability status scale (EDSS). Neurology. 1983;33(11): 1444-1452.

32. Amato MP, Ponziani G. Quantification of impairment in MS: discussion of the scales in use. Mult Scler. 1999;5(4):216-219.

33. Kümpfel T, Hoffmann LA, Rübsamen H, et al. Late-onset tumor necrosis factor receptor-associated periodic syndrome in multiple sclerosis patients carrying the Tnfrsf1a R92q mutation. Arthritis Rheum. 2007; 56(8):2774-2783.

34. Trojan DA, Arnold D, Collet JP, et al. Fatigue in multiple sclerosis: association with disease-related, behavioural and psychosocial factors. Mult Scler. 2007;13(8):985-995.

35. Hartrick CT, Kovan JP, Shapiro S. The numeric rating scale for clinical pain measurement: a ratio measure? Pain Pract. 2003;3(4):310-316.

Journal of Pain Research

\section{Publish your work in this journal}

The Journal of Pain Research is an international, peer-reviewed, open access, online journal that welcomes laboratory and clinical findings in the fields of pain research and the prevention and management of pain. Original research, reviews, symposium reports, hypothesis formation and commentaries are all considered for publication.

The manuscript management system is completely online and includes a very quick and fair peer-review system, which is all easy to use. Visit http://www.dovepress.com/testimonials.php to read real quotes from published authors.

\section{Dovepress}

\footnotetext{
(1)
} 